\title{
CompLex: an eye-movement database of compound word reading in English
}

\author{
Daniel Schmidtke ${ }^{1}$. Julie A. Van Dyke ${ }^{2}$. Victor Kuperman ${ }^{1}$
}

Published online: 22 June 2020

(C) The Psychonomic Society, Inc. 2020

\begin{abstract}
The CompLex database presents a large-scale collection of eye-movement studies on English compound-word processing. A combined total of 440 participants completed eye-tracking experiments in which they silently read unspaced English compound words (e.g., goalpost) embedded in sentence contexts (e.g., Dylan hit the goalpost when he was aiming for the net.). Three studies were conducted using participants representing the non-college-bound population (300 participants), and four studies included participants recruited from the student population (140 participants). The database comprises trial-level eye-movement data (47,763 trials), participant data (including a measure of reading experience estimated via the Author Recognition Test), and lexical characteristics for the set of 931 English compound words used as critical stimuli in the studies. One contribution of the present paper is a set of regression analyses conducted on the full database and individual experiments. We report that the most reliable and consistent main effects were those elicited by compound word length, left constituent frequency, right constituent frequency, compound frequency and semantic transparency. Separately, we also found that the effect of left frequency and compound word length is weaker among more frequent compounds. Another contribution is a power analysis, in which we determined the sample sizes required to reliably detect effect sizes that are comparable to those observed in our regression models. These sample size estimates serve as a recommendation for researchers wishing to either collect eye-movement data for compound word reading, or use the current database as a resource for the study of English compound word processing.
\end{abstract}

Keywords Megastudy $\cdot$ Eye movements $\cdot$ Compound words $\cdot$ Semantic transparency $\cdot$ Psycholinguistics $\cdot$ Morphology

\section{Introduction}

Compounding, or the linguistic process of combining lexical elements into a single word (e.g., jellyfish, raincoat, songbook), is a widely discussed topic in both linguistics, psychology of language, and language education. By its nature, compounding provides insights into how the forms and meanings of individual morphemes map onto the form and the meaning of a complex word, and what impact this word formation has on cognitive demands of learning

Electronic supplementary material The online version of this article (https://doi.org/10.3758/s13428-020-01397-1) contains supplementary material, which is available to authorized users.

Daniel Schmidtke

schmiddf@mcmaster.ca

1 McMaster University, Hamilton, Canada

2 Haskins Laboratories, New Haven, CT, USA words, and storing and retrieving them from memory when producing or comprehending language (Libben, 2006, 2014). A citation analysis of the Web of Science reference database identified 1340 papers on compound words published between 1976 and 2018. ${ }^{1}$ In this time period, papers on compounding elicited a total of 17,730 citations. The growth rate of both the publications and citations over time is superlinear.

A substantial subset of this body of literature is experiment-based and addresses production and comprehension of compound words in spoken and written modalities. These studies critically rely on both indices of linguistic behavior and quantitative estimates of orthographic, phonological, or semantic aspects of compounds and their constituent morphemes. Almost invariably, the experimental stimuli that elicit behavioral responses have been customtailored to the particular focus of a given study, and data

\footnotetext{
${ }^{1}$ The query was set to TOPIC $=$ compound word* and further manually restricted to fields in language, linguistics and psychology, to filter out the literature on chemical compounds.
} 
collection is relatively small-scale, with a few dozen of participants and items in a given experiment.

Large-scale datasets provide behavioral responses from hundreds of participants to thousands of stimuli that are constructed in a theory-independent way and have greatly contributed to the empirical base of psycholinguistic research on word processing (for an excellent review of psycholinguistic mega-studies see Keuleers \& Balota, 2015). Several papers have been specifically dedicated to the collection of norms for lexical variables related to compounds. To give a few recent examples for English compounds, Juhasz and Berkowitz (2011) reported ratings for six variables, including familiarity and lexical dominance, for 609 compound words. Furthermore, Kim et al. (2018) collected human ratings of semantic transparency (a measure of how close the meanings of a compound and its constituents are to each other), along with some additional lexical statistics, for 2861 English compound words. Semantic transparency norms are also reported for 445 English compounds in Schmidtke et al. (2018a). Furthermore, Schmidtke et al. (2018b) made available judgment data from the possible relations task collected for 604 English compound words (providing information about the likelihood of a relational meaning, e.g., "box FOR letters" for letterbox, as a possible interpretation for a compound word meaning). Conceptual relations data for a further 600 compound words is reported in Schmidtke et al. (2018c). More recently, Gagné et al. (2019) collected human semantic transparency ratings for 8304 English compound words, along with a range of other lexical characteristics, including bigram frequency, sentiment evaluated for the compound and its constituents, and the morphological family size of each of a compound's constituents.

Additionally, experimental research on compound processing has benefitted from the general-purpose collection of behavioral data from common psycholinguistic tasks: visual lexical decision, auditory lexical decision, naming and others (Balota et al., 2007; Brysbaert et al., 2016; Ernestus \& Cutler, 2015; Keuleers et al., 2012; Tucker et al., 2018). While these datasets were not designed to address compound word processing in particular, they contain behavioral responses to compound words and were used accordingly (Kuperman, 2013; Kuperman \& Bertram, 2013; Schmidtke et al., 2018b; Schmidtke \& Kuperman, 2019; Gagné et al., 2019; Schmidtke et al., 2016; Juhasz, 2018).

This paper reports a large-scale collection of eyetracking data on English sentence reading. This mega-study combines the results of seven eye-tracking experiments encompassing a total of 931 unique unspaced noun-noun compound words and 440 participants sampled either from undergraduate convenience pools or the non-university student community. A novel aspect of our database is that we provide a measure of the amount of exposure to print for individual participants in our samples, estimated via the Author Recognition Test (ART; Acheson, Wells \& MacDonald, 2008; Stanovich and West 1989). The ART measure captures the amount of reading experience in an individual and has been shown to have a robust predictive role in analyses of eye-movements during word reading and compound reading in particular, as well as response times in other lexical tasks (e.g., Choi, Lowder, Ferreira \& Henderson, 2015; Falkauskas \& Kuperman 2015; Moore \& Gordon 2015; Lowder \& Gordon 2017; Kuperman, Matsuki \& Van Dyke 2018; Schmidtke, Van Dyke \& Kuperman 2018a; discussed below).

Our interest is on unspaced, i.e., concatenated, compound word processing. The spatial layout of English compounds (i.e., whether the orthographic format of a compound is spaced, unspaced or hyphenated) is not fixed and covaries with certain lexical properties, such as the morphemic salience of a compound's constituents and the semantic relationship between the compound's constituents (Juhasz et al., 2005; Kuperman \& Bertram, 2013). Our interest in studying English unspaced compound words is that they represent established lexicalized word forms, i.e., these are orthographic forms that are entrenched in the lexical memory of the English-speaking community. ${ }^{2}$

Eye tracking is considered one of the most reliable and naturalistic paradigms for studies of reading (Liversedge et al., 2012; Rayner, 1998). We report eye-movement patterns elicited by compounds widely varying in their orthographic, phonological and semantic characteristics, elicited from native English-speaking participants of variable reading proficiency. Our first contribution then, is to supply the research field with a new empirical source of behavioral data which would enable hypothesis testing without the need for labor-intensive data collection (Keuleers \& Balota, 2015; Kuperman, 2015).

Our second contribution is to establish the impact of several lexical variables on the recognition of printed English compounds in sentence contexts. To this end, along with oculomotor measures, we publish a compilation of lexical variables drawn from multiple existing sources. Our choice of the lexical variables to consider spans from very well-established predictors of compound processing, to more elusive and under-researched ones. We briefly motivate our choices below.

Perhaps the most studied predictors are distributional, frequency-based characteristics like compound frequency, and the frequencies and family sizes of a compound's morphological constituents. ${ }^{3}$ Generally, compound words

\footnotetext{
${ }^{2}$ Eye-tracking studies that examine spaced English compound word processing include (Cutter et al., 2014; Frisson et al., 2008; Juhasz et al., 2009)

${ }^{3}$ Constituent frequencies are estimated for those constituents as standalone words. Family size of a compound's constituent is defined as a number of other compounds sharing that constituent in the same
} 
that occur more frequently and have constituents that either occur frequently as stand-alone words or as morphemes in a large number of other complex words are recognized faster (see. e.g., Baayen et al., 2010; De Jong et al., 2000). Moreover, there are reports of interactions, such that the effect of constituent frequency or family size is more pronounced in lower-frequency compounds (Kuperman et al., 2009).

Another set of predictors is relatively new and the utility of examining them can be established by pitting them against a very large dataset. The predictors in question include valence, or psychological positivity, of morphological constituents as stand-alone words: Kuperman's (2013) analysis of a lexical decision mega-study pointed to the faster recognition of compound words with more positive constituents, in line with the gradient model of automatic vigilance to negative stimuli (see review in Kuperman et al., 2014). We also examined entropy of conceptual relations, a measure of competition between possible relational interpretations for a compound word (e.g., 'ray HAS sting', 'ray CAUSES sting', and 'ray MAKES sting' as possible candidates for the compound word stingray). Schmidtke et al. (2016, 2018b) found that greater entropy of conceptual relations, i.e., greater competition between possible relations, impeded lexical decision responses in both the visual and auditory modality.

Importantly, the current dataset enables researchers to study any other lexical variable of choice using eye-tracking data on compound reading as an outcome variable. To achieve this goal, we estimated the main effect of each variable on several eye-movements, as well as interactions of those variables with one of the strongest predictors of eye-movement durations: compound frequency. These estimates are obtained from a series of mixed-effects regression models and reported in summary tables below.

The expansion in availability of both behavioral data serving as dependent variables, and data on lexical measures that serve as independent variables affords a possibility that individual experimental studies lack, i.e., a multi-fold increase in sample size and power of statistical inference. With this in mind, our third contribution is to estimate the sample size that is required for an individual experiment that aims at testing an effect of a given magnitude. Together with our second goal, the outcome of this step is to determinebased on the largest dataset of its kind yet-whether the pursuit of some of the more controversial lexical effects discussed in the literature (e.g., semantic transparency) is worthwhile given the practical limitations of sample-size in any given experiment.

position (Schreuder \& Baayen, 1997), e.g., the family of post includes postman, post office, postbag etc.

\section{Methods}

\section{Participants}

A total of 440 participants contributed to the database by participating in one of seven experimental studies reported below. All were native speakers of English, did not have a diagnosed visual or learning disability, and had normal or corrected-to-normal vision. Participants for each specific study were recruited either from the community (Hamilton, Ontario, Canada or New Haven, Connecticut, USA) or from the McMaster University convenience pool of undergraduate students. All New Haven-based studies were approved by the Yale University Institutional Review Board. All McMaster-based studies were approved by the local McMaster Research Ethics Board (McMaster University). Below we describe the recruitment procedure and compensation for both types of cohorts.

\section{Community population samples}

Study 1 One hundred thirty-four participants (79 female; 50 male; five undisclosed) were recruited in New Haven, Connecticut, within an age range of $16-26(\mathrm{M}=20.57$, SD $=2.19$ ). Participants were paid $\$ 15 \mathrm{USD} / \mathrm{h}$ and recruited from the local community in a number of ways, including presentations at adult education centers; advertisements in local newspapers; posters/flyers placed on adult school and community college campuses, public transportation hubs, local retail and laundry facilities; and from referrals from past and current study participants. All participants were non- college-bound individuals (formal level of education did not exceed the equivalent of high school level). A subset of this data is reported in Schmidtke et al. (2018a).

Study 2 One hundred twenty-one participants (66 female; 55 male) were recruited in New Haven, Connecticut, USA, within an age range of $16-26(\mathrm{M}=21.19, \mathrm{SD}=2.32)$. The recruitment, payment and educational profile of participants in this study was the same as in Study 1.

Study 3 Forty-five participants ( 23 female; 22 male) were recruited in Hamilton, Ontario, Canada, within an age range of $18-31(\mathrm{M}=23.24, \mathrm{SD}=4.21)$. Participants were paid \$15-20 CAD/h and were recruited from the local community in a number of ways, including presentations at local colleges; advertisements placed on local community sections of online classified advertising services (Craigslist, Indeed, and Kijiji); posters/flyers placed on adult school and community college campuses, public transportation hubs, and from referrals from past and current study participants. All participants were non-college-bound individuals (formal 
level of education did not exceed the equivalent of high school level).

\section{University population samples}

Study 4 Twenty-six undergraduate students from McMaster University (21 female; five male) within an age range of 17$28(\mathrm{M}=19.96, \mathrm{SD}=2.65)$ completed the eye-tracking study for course credit. All participants were native speakers of English.

Study 5 Thirty-five undergraduate students from McMaster University (26 female; nine male) within an age range of 18-37 $(\mathrm{M}=20, \mathrm{SD}=3.58)$ completed the eye-tracking study for course credit.

Study 6 Thirty-eight undergraduate students from McMaster University ( 27 female; 11 male) within an age range of 18-30 $(\mathrm{M}=20.82, \mathrm{SD}=2.7)$ completed the eye-tracking study for course credit.

Study 7 Forty-one undergraduate students from McMaster University (34 female; six male; one undisclosed) within an age range of $18-26(\mathrm{M}=19.98, \mathrm{SD}=1.79)$ completed the eye-tracking study for course credit.

Studies 3, 4, 5, 6, and 7 were reported in Schmidtke and Kuperman (2019).

\section{Apparatus}

Eye-movements in all experiments were recorded with an EyeLink 1000 eyetracker, manufactured by SR Research Ltd. (Kanata, Ontario, Canada). The eyetracker is an infrared video-based tracking system combined with hyperacuity image processing. The eye-movement camera and a conjoined infrared illuminator were mounted on a desktop beneath the stimulus display. The recording was monocular (right eye). The camera sampled pupil location and pupil size at a rate of $1000 \mathrm{~Hz}$. A chin support and forehead rest was used to stabilize participants' gross head movements. The stimuli were presented on a 10.75-in x 13.25-in screen with a refresh rate of $60 \mathrm{~Hz}$. The average gaze position error of the EyeLink 1000 is $<.05^{\circ}$, while its resolution is $.01^{\circ}$ (root mean square error), with a micro-saccade resolution of $.01^{\circ}$.

\section{Materials}

In all experiments, a trial consisted of a sentence frame containing one English concatenated noun-noun compound word (The stain on the workbench was silvery and gross.). The compound words were extracted from the CELEX database (Baayen et al., 1995) and the ELP lexical database
(Balota et al., 2002). A small number of compounds were used in more than one sentence frame. The total number of sentences with compounds across all experiments was 978 and the number of unique compounds was 931 .

Table S1 in Supplementary materials reports the number of observations for each compound in the full database and for each individual experiment. In some experiments, only sentences containing compounds were presented; in others, sentences with compounds served as fillers for trials with a different syntactic manipulation. The data considered here is comprised of sentences with only compounds and no additional manipulation. The dataset contains 5 different stimuli lists containing a different number of sentence frames with different compound words (List $1=139$; List $2=215$; List $3=213$; List $4=200$; List $5=198$ ). Some lists were used for multiple experiments and some stimuli lists were intermixed for certain experiments. Table S2 in Supplementary materials reports the distribution of stimuli lists across each experiment.

All sentences were constructed such that the compound word never occupied the first or the last position in the sentence. The context up to the word after the compound was kept neutral, i.e., did not provide a strong cue towards the identity of the target word, as demonstrated in additional norming studies. All compounds were unspaced and plausible in their contexts, as established through additional norming studies. Every sentence was restricted to at most 90 characters and occupied exactly one line on the screen.

All sentences were displayed in Arial 14 point font. The height for the font was $.5 \mathrm{~cm}$ and was viewed at a distance of $60 \mathrm{~cm}$, where one character space subtended approximately $.48^{\circ}$ of visual angle. To track the performance of participants, in some experiments, comprehension questions would appear after certain trials, which required a "yes" or "no" response. Where administered, comprehension questions were asked after $25-30 \%$ of critical trials. The order of sentences was pseudo-randomized for each participant in a given experiment. Each experimental session began with a series of $4-8$ practice trials.

\section{Procedure}

Prior to presentation of the stimuli, in all experiments the eye-tracker was calibrated using a series of nine fixed targets distributed around the display, followed by a ninepoint accuracy validation. Calibration was repeated after any breaks or whenever the experimenter judged necessary. Participants were instructed to read each sentence silently for comprehension and were told that they would be required to answer a comprehension question after some trials. Participants were told that they could take a break at any point during the experiment. Each trial began with 
a screen containing a fixation point in the middle left of the screen. While fixating on this point, the experimenter pressed a button which would display a sentence on the screen. Participants were limited to $10 \mathrm{~s}$ to complete the reading of the stimulus sentence.

After they had read the sentence, participants were instructed to look at a dark rectangle at the bottom right corner of the screen, which triggered a comprehension question to appear (e.g., for the sentence The bookshelf was broken during the burglary last night. the associated comprehension question was Did anything break during the burglary?). The question appeared in the center of the screen; two possible answers appeared three lines below, one to the left of center and one to the right of center. Participants indicated their answer by pressing buttons on the left and right side of the keyboard which were associated with the left and right sides. The position of the correct answer was counterbalanced throughout the experiment. If participants had not signaled that they had completed reading the sentence within the 10 -second limit, the computer moved onto the comprehension question automatically. Participants were told to make their best guess at the comprehension question if they were unsure of the answer. If they had not answered within a 10-second limit, the computer moved onto the next item.

\section{Databases}

CompLex comprises three linked databases, in commaseparated . CSV format. One is a database of participant information, CompLexParticipantData.Csv, which includes reader characteristics, such as information about the study that each of the participants belonged to, as well as basic demographic information and individualdifferences measures. A second one is a database of compounds, CompLexLexicalData.csv, which provides information, including lexical statistics, about each target word and its corresponding carrier sentence frame. A third database, CompLexEyeMovementData.Csv reports basic eye-movement measures for the target word in each sentence frame per each experiment. Finally, a master file CompLexCombined.CsV merges together all information using two key fields: SubjectID and SentenceFrameID.

\section{Reader characteristics}

This database reports the target population for recruitment (Community vs. University) (PopulationSample), age (Age), and sex of the participant (Sex) in each study, along with their participant and study IDs (SubjectID and Study). Additionally, it reports the participant score in the Author Recognition Test (ART), a test that measures an individual's amount of exposure to printed materials (Stanovich \& West, 1989).

We administered an updated version of Stanovich and West (1989) ART Acheson et al. (2008). In the ART, participants are presented with an intermixed list of 130 real and false author names from the modern literature printed on a single sheet of paper: 65 names are real authors and the remaining 65 names are distractors. Participants were instructed to only mark those names that belong to authors. They received one point for every correctly identified author and lost one point for every incorrect identification. The underlying assumption is that-through extensive reading-readers either read works by these authors or are exposed to their names in other literature. ${ }^{4} \mathrm{~A}$ possible score range is from -65 to 65 . A score of 0 means that they had as many correct responses as incorrect ones. A total of 426 participants ( $97 \%$ of the entire sample) were administered the ART.

Finally, for some studies, comprehension scores are available: these scores represent participant-level accuracy in responding to comprehension questions.

Table 1 reports descriptive statistics of the reader characteristics in the combined datasets. A summary by individual experiment is available in Table S3 in Supplementary materials.

\section{Compound word characteristics}

One of our goals is to estimate the effect of multiple lexical variables on reading times to English noun-noun compounds, see Introduction. Most of the variables identify the compound and its left and right constituent morphemes (typically, the modifier and the head in English) and carry the prefix Compound, Left and Right, respectively. The variables Compound, LeftConstituent, RightConstituent compound word, its immediate constituent morphemes. The columns sentence and SentenceFrameID report the carrier sentence frame for the compound; WordPosition additionally specifies the position that the target compound occupied in the sentence. The variable List provides the experimental list number.

\footnotetext{
${ }^{4}$ There is evidence to suggest that the ART is a valid test for younger readers, i.e., readers in our sample. In an item response theory analysis, Moore and Gordon (2015) show that item difficulty is predicted by the frequency of occurrence of authors on Internet webpages. Author names that were more frequently attested in the Google Terabyte NGram Corpus of English Web sites (one trillion tokens) tended to be correctly identified more often than less frequently attested author names. This finding may suggest that an individual's success in the ART may be partly explained by the amount of that individual's cultural knowledge that is acquired from experience with internet texts.
} 
Table 1 Summary of reader characteristics in the combined dataset

\begin{tabular}{llllll}
\hline Participant characteristic & $n$ & Mean & SD & Median & Min \\
\hline Age & 434 & 20.89 & 2.78 & 21.00 & 16.00 \\
ART & 426 & 11.30 & 8.88 & 9.00 & 37.00 \\
Comprehension & 185 & 90.55 & 5.27 & 91.00 & 4.00 \\
\hline
\end{tabular}

Variables ending in Length (e.g., CompoundLength) report orthographic lengths (in characters) of the respective word and constituents. Variables ending in Frequency (e.g., LeftFrequency) report frequencies of occurrence of the compound and its constituents as stand-alone words in the 51 million-token SUBTLEX-US corpus of subtitles to the US films and media (Brysbaert \& New, 2009). Variables ending in Familysize report counts of complex English words that share either the left or the right constituent with the target compound, respectively. These counts were extracted from the CELEX database (Baayen et al., 1995). Variables ending in Valence represent average ratings of valence (or psychological positivity on the scale from 1-unpleasant to 9-pleasant) for the compounds and their constituents as stand-alone words: the ratings come from Warriner et al. (2013) database of affective norms.

We further included two types of semantic transparency estimates. One is based on latent semantic analysis (LSA; Landauer \& Dumais 1997) which provides computational estimates of distance between words represented as cooccurrence vectors in a multidimensional semantic space. The degree of semantic similarity between the words that those vectors represent is estimated by the cosine of the angle between the vectors, ranging from 0 to 1 . We inverted the raw scores (by multiplying by negative one) that were collected from the web interface for comparability with other LSA scores. After the inversion, values closer to 1 imply a greater semantic similarity between the pair of words under comparison. The term-to-term LSA scores were collected for three pairs formed by the compound and its constituents as stand-alone words: a comparison between the left and the right constituents (apple vs. sauce); the left constituent and the compound (apple vs. applesauce); and the right constituent and the compound (sauce vs. applesauce). The estimates were collected from the online tool http://meshugga.ugent.be/snaut-english/ with a default setting of 300 factors and a window of six words (Mandera et al., 2017). These LSA scores were calculated over word occurrences in the 51 million-token SUBTLEX-UK corpus of film subtitles (van Heuven et al., 2014). These semantic similarity ratings carry the prefix LSA (e.g., LSALeftCompound for the semantic similarity between the standalone left constituent and the compound word).

Another set of estimates are human ratings of semantic similarity between pairs of words. These ratings are available for the relationship between the left constituent and the compound (apple vs. applesauce), and the right constituent and the compound (sauce vs. applesauce). The semantic transparency ratings for the semantic similarity between the left constituent and the compound word is labelled as LeftST and for the semantic similarity between the right constituent and the compound word is named RightST. We included two sets of human semantic transparency ratings for compound words in our database. The first is the set of ratings reported in Schmidtke et al. (2018a), which are identified with the suffix _SVDK. We also report the human ratings reported in Kim et al.'s (2018) database of human ratings of semantic transparency. Kim et al.'s (2018) ratings were included because the compounds used in their study currently provide the largest available overlap with the list of compounds in the present database $(N=882)$. These ratings are identified with the suffix _KYG. Kim et al. (2018) defined semantic transparency as "the degree of relatedness in meaning between a compound word and its constituents" (pp. 1). These ratings are based on how participants rated the strength of the relationship between the meanings of a compound word and either its first or second constituent (e.g., cheeseburger - cheese or cheeseburger - burger). These ratings used a 1 -to-7 scale $(1=$ not related, $7=$ highly related $)$. Different operationalizations of semantic transparency exist beyond the distinction between human and computational norms, such that there are multiple ways in which human transparency ratings are defined and collected (for excellent discussions of this issue, see Gagné et al., 2019 and Kim et al., 2018).

The next set of reported variables concerns the nature and diversity of conceptual relations which link the meanings of a compound's constituents. This data comes from two experiments for which a detailed procedure and raw data are available in Schmidtke et al. (2018b and c). In these experiments, participants were presented with a compound word (e.g., applesauce) and were asked to choose one relational interpretation that they think is the most likely literal meaning out of a set of 16 possible relational interpretations (e.g., 'sauce CAUSES apple', 'sauce CAUSED BY apple', 'sauce HAS apple', 'sauce BY apple', 'sauce MAKES apple', 'sauce FROM apple', 'sauce MADE OF apple', 'sauce IS apple', 'sauce USED BY apple', 'sauce USES apple', 'sauce LOCATED apple', 'apple LOCATED 
sauce', 'sauce FOR apple', 'sauce ABOUT apple', 'sauce DURING apple', and 'sauce BY apple'). We report the selection frequency of each relational interpretation per each compound, e.g., for the compound applesauce 'sauce MADE OF apple' was the most frequently chosen option, since 27 participants out of 49 chose this as the most suitable relational meaning. The selection frequencies are each reported in separate columns, which are labelled with the conceptual relation (e.g., made_of) along with the head $(\mathrm{H})$ and modifier $(\mathrm{M})$ either as a prefix or suffix depending on the relation, e.g., 'sauce MADE OF apple' is H_made_of_M. We also report Shannon entropy calculated over the probability distribution of interpretations of conceptual relations for a given compound. Entropy of conceptual relations (EntropyConceptRel) is defined as $H=-\Sigma p_{i} \log _{2} p_{i}$, where $p_{i}$ is the probability of a relation within the respective distribution of chosen possible relations. This measure was estimated only for the set of conceptual relations selected more than once for a given compound. As stated above, this measure is an operationalization of competition between cogenerated conceptual relations during lexical processing and is predictive of auditory and visual lexical decision responses to compound words. Greater entropy, i.e., greater competition, is linearly associated with longer lexical decision responses (see Schmidtke et al., 2018b).

Finally, all of the experiments were accompanied with norming studies evaluating plausibility of the compound in its context (the description of these ratings studies are provided in the Supplementary materials of Schmidtke et al., 2018a). The plausibility ratings (MeanPlausibility) range from 1 (very implausible) to 5 (very plausible): average values are reported. For some experiments, we collected close predictability ratings (ClozeProb). Cloze predictability is defined as the proportion of raters who identified the target word correctly based on the preceding context in a sentence completion task and ranges from 0 to 1 . The Cloze predictability values were zero for $100 \%$ of compounds. This is a verification of a requirement of our stimuli design which was that target compounds are unpredictable from their context. Missing values for cloze predictability ratings are coded as NA.

Beyond the processing constraints of the plausibility and predictability of the target word in the sentence (for reviews, see Schotter, Lee, Reiderman \& Rayner 2015; Staub, Rayner, Pollatsek, Hyönä \& Majewski 2007; Staub $2015)$ the eye-movement literature reports effects on the eye-movements to a word that do not come from the properties of that word. These include the effects of length and frequency of the pre- and post-target words (for a review see Rayner, 2009). However, since these effects are both conceptually and statistically orthogonal to the goal of the present paper, we do not include these variables in our regression models in the present study. The published dataset includes carrier sentences (see above) which will enable researchers to obtain these measures directly if they wish to include them in regression models.

Table 2 reports descriptive statistics of the lexical characteristics in the combined datasets, including the total number of unique observations for each lexical variable. A summary by individual experiment is available in Table S4 in Supplementary materials.

\section{Eye-movement variables}

The following eye-movement measures are included in the report for every target word: first fixation duration (labeled FFD), second fixation duration (SECFD); gaze duration (GD, the summed duration of all fixations on the word before the gaze leaves the word for the first time); total fixation time (TFT, the summed duration of all fixations on the word); landing position of the first fixation on the word ( $\mathrm{LP}$, in characters: 0 stands for the space before the word); regression path duration ( $R P D$, the summed duration of all fixations on the target word and to the left of the word, before the gaze leaves the word in the direction of reading for the first time); selective regression path duration (SRPD, the summed duration of all fixations on the target word before the gaze leaves the word in the direction of reading for the first time); regression in (REGIN, a binary measure showing whether the gaze regressed to a word from another word further in the sentence), regression out (REGOUT, a binary measure showing whether the gaze regressed from the word to another word towards the beginning of the sentence); fixation count (FIXC, total number of fixations on a word); refixation (REFIX, a binary measure indicating whether a word was fixated more than once in the first pass of reading); and skipping (SKP, a binary indicator of whether the word was skipped). For all variables, values are set to NA if they are not observed. All durational measures in the database are on the millisecond scale. Additionally, we report the IDs of the participant (SubjectID) and the sentence (SubjectFrameID), as well as the ordinal number of the trial in which the sentence was presented to the reader in a given experiment (TrialNumber).

Data cleaning procedures With all studies compiled, the initial raw data set consisted of 49,792 trials for which the eyetracking signal was not lost on the critical compound word in the sentence (but may have been lost in other parts of the sentence). We removed the top $1 \%$ of the distribution of fixation counts, a measure which includes fixations which occurred as a consequence of refixations and regressions on the compound word. This equated to removing trials that were fixated on more than six times. We also removed 
Table 2 Summary of lexical characteristics in the full database

\begin{tabular}{|c|c|c|c|c|c|c|}
\hline Lexical variable & $n$ & Mean & SD & Median & Min & Max \\
\hline CompoundLength & 931 & 8.57 & 1.26 & 9.00 & 6.00 & 14.00 \\
\hline CompoundFrequency & 931 & 104.58 & 339.82 & 30.00 & 0.00 & 4958.00 \\
\hline LeftFrequency & 931 & 6676.88 & 12616.19 & 2923.00 & 9.00 & 133117.00 \\
\hline RightFrequency & 931 & 12891.74 & 24524.19 & 3383.00 & 0.00 & 204428.00 \\
\hline LeftFamilySize & 931 & 9.86 & 10.53 & 6.00 & 0.00 & 54.00 \\
\hline RightFamilySize & 931 & 18.89 & 28.66 & 10.00 & 0.00 & 155.00 \\
\hline LeftValence & 914 & 5.68 & 1.14 & 5.75 & 1.83 & 8.34 \\
\hline RightValence & 898 & 5.64 & 0.98 & 5.65 & 1.55 & 7.82 \\
\hline LSALeftRight & 930 & 0.26 & 0.11 & 0.24 & -0.03 & 0.79 \\
\hline LSALeftCompound & 921 & 0.30 & 0.13 & 0.29 & -0.00 & 1.00 \\
\hline LSARightCompound & 920 & 0.29 & 0.13 & 0.28 & -0.04 & 0.64 \\
\hline LeftST_SVDK & 785 & 4.45 & 1.01 & 4.50 & 1.86 & 6.77 \\
\hline RightST_SVDK & 785 & 4.37 & 0.98 & 4.36 & 1.40 & 6.79 \\
\hline EntropyConceptRel & 903 & 2.00 & 0.62 & 2.05 & 0.00 & 3.31 \\
\hline
\end{tabular}

all trials for which a fixation on the critical compound word lasted for less the $50 \mathrm{~ms}$ (698 trials). These are fixations are likely due to oculomotor programming rather than cognitive processing (Morrison, 1984). We removed any fixations on compound words longer than $1000 \mathrm{~ms}$, gaze durations longer than $1200 \mathrm{~ms}$, and total fixation times longer than $1500 \mathrm{~ms}$ (660 data points altogether; all upper thresholds were set to cut off the top $1 \%$ of each duration distribution). The final published eye-movement database contains 47,763 trials, and includes observations where the compound word was skipped. Table 3 reports descriptive statistics of the eye-movement measures in the combined datasets. A summary by individual experiment is available in Table S5 in Supplementary materials.

In what follows, we discuss three duration measures that have been shown to reflect the time-course of compound processing: first fixation duration, gaze duration, and total fixation time.

\section{Statistical considerations}

We conducted three kinds of analyses. We first present an analysis of main effects of a select set of lexical variables (see Methods) on the eye-movement record of compound word reading. Next we present an analysis of interactions of those lexical variables with compound frequency as predictors of eye-movements to compound words. Our focus on compound frequency is motivated by robustness of this effect on production and comprehension of compounds (Baayen et al., 2010) and earlier findings of the interactions between this frequency measure and multiple other lexical variables (see the Introduction). Compound frequency can be interpreted as a measure of a compound's entrenchment in the mental lexicon (see discussion in Schmid, 2010): its interactions with other variables reveal which properties of a compound and its morphemes induce processing effort when the compound is largely lexicalized or, conversely,

Table 3 Summary of eye-movement characteristics in the full database

\begin{tabular}{|c|c|c|c|c|c|c|}
\hline Eye-movement variable & $n$ & Mean & SD & Median & Min & Max \\
\hline LP & 46473 & 49.51 & 32.62 & 45.60 & 0.00 & 239.10 \\
\hline FFD & 46473 & 234.36 & 87.24 & 218.00 & 51.00 & 988.00 \\
\hline REFIX & 47763 & 0.39 & 0.49 & 0.00 & 0.00 & 1.00 \\
\hline SECFD & 30147 & 209.28 & 89.23 & 192.00 & 51.00 & 968.00 \\
\hline GD & 46473 & 337.01 & 181.01 & 289.00 & 51.00 & 1199.00 \\
\hline REGOUT & 46473 & 0.23 & 0.42 & 0.00 & 0.00 & 1.00 \\
\hline RPD & 46473 & 440.13 & 307.13 & 360.00 & 51.00 & 5049.00 \\
\hline SRPD & 46473 & 376.16 & 202.74 & 329.00 & 51.00 & 1499.00 \\
\hline REGIN & 46473 & 0.24 & 0.43 & 0.00 & 0.00 & 1.00 \\
\hline TFT & 47763 & 449.13 & 266.43 & 392.00 & 0.00 & 1499.00 \\
\hline FIXC & 47763 & 2.05 & 1.16 & 2.00 & 0.00 & 6.00 \\
\hline
\end{tabular}


relatively unfamiliar. Finally, we present a power analysis for lexical effects of varying magnitudes, see below.

The first two sets of analyses were conducted both on the full database and on individual experiments. The following lexical variables were selected: CompoundFrequency, LeftFrequency, RightFrequency, LeftFamilySize, Right FamilySize, CompoundLength, LeftValence, RightValence, EntropyConceptRel, LeftST, and RightST (in analyses we used the human semantic transparency ratings reported in Kim et al. (2018) since these ratings provide the greatest overlap with the compounds in the current database). Other lexical variables contained too many missing values. As an individual-difference independent variable we considered ART. Finally, we only concentrated on durational eye-movement measures as dependent variables: first fixation duration (FFD), gaze duration (GD), and total fixation time (TFT). If other variables are of interest to our readers, they are invited to conduct additional analyses using our database.

We started out with the full cleaned database of 47,763 observations. We removed all observations for which the compound word was skipped (1290 trials) and all trials for which there were any missing values for the lexical variables of interest or for participants who did not have an ART score (7802 trials). The final dataset for the current analysis contained 38,671 valid trials.

Both of these sets of analyses consisted of a series of linear mixed-effects multiple regression models. The first set of analyses examined main effects of the set of lexical predictor variables. Each model in these analyses had one of the three dependent variables (first fixation duration, gaze duration or total fixation time). As independent variables, the model included the entire set of 11 lexical variables plus ART. As a control variable we included MeanPlausibility as a fixed effect. We also included by-subject and by-item random effects. Additionally, each model had one of the lexical independent variables included in the random-effects structure. The generic formula for a regression model was:

$$
\begin{aligned}
& D V \sim \text { Lex }_{i}+A R T+\text { Mean Plausibility } \\
& +(1 \mid \text { Compound })+\left(1+\text { Lex }_{i} \mid \text { Subject } I D\right),
\end{aligned}
$$

where DV is the dependent variable, Lex is a set of $n$ lexical predictors, and $\operatorname{Lex}_{i}$ is the critical predictor of choice from the set of variables in Lex. For instance, one model would contain 11 independent variables including CompoundLength as predictors of first fixation duration, along with the by-item random intercepts, by-subject random intercepts, by-subject random slopes of CompoundFrequency and the correlation between the by-subject random intercepts and slopes. In comparison, another model would have the same structure except RightFrequency would be included as by-subject random slopes. A total of 33 models (11 lexical predictors $\mathrm{x} 3$ dependent variables) were fitted to the full dataset and the same number of models were fitted to data for each individual experiment.

The statistical procedure was very similar in the analysis where interactions with compound frequency were examined. Ten lexical predictors (all except compound frequency) were allowed to enter into an interaction with compound frequency in separate models. Initially, the entire interaction was included in the random effects structure as by-subjects slopes. Since most such models failed to converge, the random slopes were simplified by only incorporating the by-subject random slopes of the variable which interacts with CompoundFrequency (e.g., LeftValence). The trimming procedures and the cut-off threshold of the $t$-value for the critical interaction were as described above. Regression models in this set took the following generic formula:

$$
\begin{aligned}
& D V \sim \text { Lex }_{-i}+A R T+\text { Lex }_{i} * \text { CompoundFrequency } \\
& + \text { MeanPlausibility }+(1 \mid \text { Compound }) \\
& +\left(1+\text { Lex }_{i} \mid \text { Subject I } D\right),
\end{aligned}
$$

where DV is the dependent variable, and $\operatorname{Lex}_{-i}$ is a set of $n-1$ lexical predictors, which excludes the critical predictor of interest $\mathrm{Lex}_{i}$. A total of 30 models (10 lexical predictors $\mathrm{x} 3$ dependent variables) were fitted to the full dataset and for each of the individual experiments.

Dependent variables were log-transformed to attenuate the influence of outliers. ${ }^{5}$ Similarly, all frequency-based lexical predictors (frequency and family size) were logtransformed prior to modeling. For each analysis, we refitted the final model after removing outliers from both data sets by excluding standardized residuals exceeding -2.5 or +2.5 standard deviations. The package lme4 (version 1.1-19) in the $\mathrm{R}$ statistical software was used for regression modeling. We extracted $t$-values for the critical predictor from each model (e.g., CompoundLength or RightFrequency). Many of our variables are highly correlated with one another (see

\footnotetext{
${ }^{5}$ Following Schramm and Rouder (2019) recent investigation of the appropriacy of analyzing log-transformed response time data, we conducted all analyses without log-transforming the dependent variables. We found that all of the effects observed with logged durational measures were also found when predicting raw nontransformed measures. This was the case for effects in the full database and across individual experiments. Our motivation for examining logtransformed variables was that all durational measures were positively skewed. These distributions violate the normality assumption of linear regression models. We wanted to keep in check the overly influential values in our empirical distributions that may bias model fit. Though Schramm and Rouder present arguments against the transformation of response time measures when fitting statistical models, the reason for our choice of using logged response times is that this procedure is in keeping with the current convention of the field. If best practices in the field change, then future studies using the current database will be able to model compound word reading without log transforming durational eye-movement measures (or performing any other corrective transformation).
} 
Table 4). Following Tomaschek et al. (2018) we assessed the severity of multicollinearity in our models using the variance inflation factor (VIF). A VIF score of $>10$ for a predictor signifies that its model coefficient is poorly estimated (Kutner et al., 2004). We obtained VIFs in R using the vif () function in the car package (Fox et al., 2012). We found that the variance inflation factor did not exceed 2 for all of our predictors. For the present data, it is clear that multicollinearity does not pose a problem for model estimation.

Finally, as suggested by von der Malsburg and Angele (2017) we applied two type I error rate corrections to the results. Our data analysis produces three effects (i.e., FFD, GD and TFT) for each lexical variable (either as a main effect or in an interaction with compound frequency). We define these three effects as a family and the probability of discovering two significant effects with $p$ values $<.05$ is .0073. The first family-wise rule-of-thumb correction relies on this probability. We therefore count an effect as statistically reliable if there were at least two effects out of three at $p<.05$ in a family of models. Second, using the false discovery rate method (Benjamini \& Hochberg, 1995), raw $p$ values were adjusted in order to control for the false-positive rate by providing expected proportion of false-positives among all positive test decisions. We applied both type I error rate criteria independently: an effect qualified as a true rejection of the null hypothesis if at least one criterion was satisfied.

\section{Results and discussion}

Descriptive statistics of the dependent variables, including sample size, are reported in Table 3. Table 4 shows correlations between lexical variables and eye-movement measures in the database.

\section{Main effects}

Our first analysis assessed main effects of lexical predictors on durational eye-movements. For readability, we only report the slope estimates of lexical predictors for the full dataset. Statistical significance at the 5\% level (after application of multiple comparisons corrections) is reported as an asterisk for both the full dataset and individual experiments. This choice of presentation format does not imply that we ascribe a special importance to the nominal threshold of statistical significance: we simply view it as an indicator by which to compare between models for individual experiments and those for the full compiled database. Table 5 displays the results of this analysis, including the slope estimates from the full models for each eye-movement measure.
Regression models fitted to the full dataset revealed robust main effects of compound frequency, left constituent frequency and right constituent frequency. These effects were negative, as expected: more frequent compounds and compounds with more frequent constituent morphemes were processed faster. Moreover, there were main effects of compound length. Longer compounds were processed longer: this positive effect was robust in both the full set and individual experiments. The effect of compound length was not observed in first fixation duration: this early null effect is in line with prior reports that the influence of word length (in complex or simplex words) unfolds in later eye-movement measures (Kuperman \& Van Dyke, 2011; Kuperman et al., 2018). Furthermore, measures of semantic transparency had a facilitatory effect on processing effort: greater similarity between the left constituent and the whole compound (LeftST) was associated with shorter gaze durations and total fixation times, while greater similarity between the right constituent and the whole compound (RightST) reduced all eye-movement durations (see Schmidtke et al. 2018a for similar main effects of semantic transparency on the eye-movement record).

Other lexical predictors either showed sporadic effects in the full dataset (e.g., compounds with a left constituent carrying a more positive meaning, such as joyride, were associated with shorter gaze durations), or no reliable effect at all (e.g., RightFamilySize, or EntropyConceptRel). Notably, some of these null effects emerge as statistically reliable in one or more individual experiments (e.g., LeftFamilySize). This discrepancy illustrates an oft-made point that underpowered experiments may lead to spurious and inflated effects (Ioannidis, 2005; Button et al., 2013).

\section{Interactions with compound frequency}

This analysis allowed all lexical variables of interest (except compound frequency) to interact with compound frequency. Table 6 reports the results: asterisks mark models in which the critical interaction reached statistical significance at the 5\% level (after application of multiple comparisons corrections). Only a few such variables demonstrated reliable interactions, and all interactions were of a similar nature. Specifically, the influence of a lexical variable was the strongest in lower-frequency compounds and the weakest in higher-frequency compounds. For example, relatively frequent compounds showed a weaker positive effect of compound length on all dependent variables, a trend which is in line with studies of simplex word reading (e.g., Hyönä and Olson 1995; Rau, Moeller \& Landerl 2014; Tiffin-Richards \& Schroeder 2015). Higherfrequency compounds also showed weaker negative effects of left constituent frequency on all dependent variables (see 


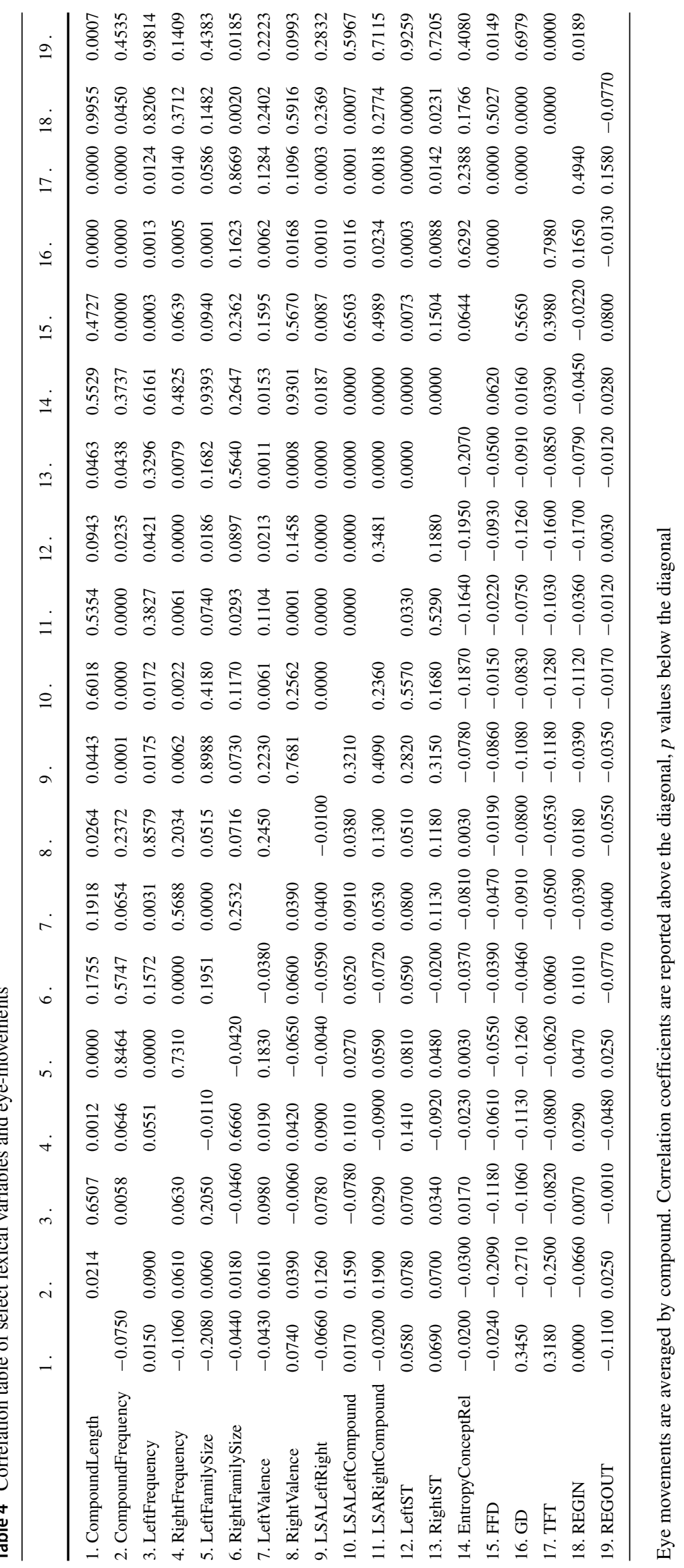


Table 5 Summary of main effects of lexical predictors in the full dataset and across individual experiments

\begin{tabular}{|c|c|c|c|c|c|c|c|c|c|c|}
\hline Eye-movement variable & Predictor & Slope & Full & Study_1 & Study_2 & Study_3 & Study_4 & Study_5 & Study_6 & Study_7 \\
\hline First fixation duration & EntropyConceptRel & 0.002 & & & & & & & & \\
\hline Gaze duration & EntropyConceptRel & -0.001 & & & $*$ & & & & & \\
\hline Total fixation time & EntropyConceptRel & 0.003 & & & & & & & & \\
\hline First fixation duration & LeftValence & -0.005 & & & & & & & & \\
\hline Gaze duration & LeftValence & -0.016 & $*$ & & & & & & $*$ & \\
\hline Total fixation time & LeftValence & -0.013 & & & & & & & $*$ & \\
\hline First fixation duration & RightValence & -0.003 & & & & & & & & $*$ \\
\hline Gaze duration & RightValence & -0.011 & & & & & & & & $*$ \\
\hline Total fixation time & RightValence & -0.014 & & & & & & & & \\
\hline First fixation duration & LeftFamilySize & -0.001 & & & & & & & $*$ & \\
\hline Gaze duration & LeftFamilySize & -0.013 & & & & & $*$ & $*$ & & \\
\hline Total fixation time & LeftFamilySize & -0.010 & & & & & & $*$ & & \\
\hline First fixation duration & RightFamilySize & -0.002 & & & & & & & & \\
\hline Gaze duration & RightFamilySize & -0.015 & & & & & & & & \\
\hline Total fixation time & RightFamilySize & -0.011 & & $*$ & & & & & & \\
\hline First fixation duration & LeftFrequency & -0.010 & $*$ & & & & & & $*$ & \\
\hline Gaze duration & LeftFrequency & -0.030 & $*$ & & & & $*$ & & & \\
\hline Total fixation time & LeftFrequency & -0.027 & & & & & & & & \\
\hline First fixation duration & RightFrequency & -0.008 & & & & & & $*$ & & \\
\hline Gaze duration & RightFrequency & -0.031 & $*$ & $*$ & $*$ & & & $*$ & & \\
\hline Total fixation time & RightFrequency & -0.035 & $*$ & & & & & & & \\
\hline First fixation duration & CompoundFrequency & -0.028 & $*$ & $*$ & $*$ & $*$ & $*$ & $*$ & $*$ & $*$ \\
\hline Gaze duration & CompoundFrequency & -0.083 & $*$ & $*$ & $*$ & $*$ & $*$ & $*$ & $*$ & $*$ \\
\hline Total fixation time & CompoundFrequency & -0.117 & $*$ & $*$ & $*$ & $*$ & $*$ & $*$ & $*$ & $*$ \\
\hline First fixation duration & CompoundLength & -0.003 & & & & & & & & \\
\hline Gaze duration & CompoundLength & 0.050 & $*$ & $*$ & $*$ & $*$ & $*$ & $*$ & $*$ & $*$ \\
\hline Total fixation time & CompoundLength & 0.059 & $*$ & $*$ & $*$ & $*$ & $*$ & $*$ & $*$ & $*$ \\
\hline First fixation duration & LeftST & -0.008 & & & & & & & & \\
\hline Gaze duration & LeftST & -0.020 & $*$ & & $*$ & & $*$ & $*$ & & \\
\hline Total fixation time & LeftST & -0.028 & $*$ & $*$ & $*$ & $*$ & $*$ & $*$ & $*$ & \\
\hline First fixation duration & RightST & -0.010 & $*$ & & $*$ & & & & & \\
\hline Gaze duration & RightST & -0.025 & $*$ & $*$ & $*$ & & & $*$ & & \\
\hline Total fixation time & RightST & -0.041 & $*$ & $*$ & $*$ & & & & & \\
\hline
\end{tabular}

\footnotetext{
* indicates the presence of a main effect with $t>2$ after the application of multiple comparison correction criteria. Slope estimates from linear mixed effects models are provided for the full dataset
}

Kuperman et al., 2008, 2009). In all cases, the difference in fixation times between the extreme values of a given lexical predictor (e.g., the shortest and longest compounds) was smaller in higher-frequency compounds than in lowerfrequency ones. Figure 1 depicts the partial effects of left constituent frequency on gaze duration (panel A), and compound length on total viewing time (panel B), each broken down by 10th, 30th, 50th, 70th, and 90th percentiles of compound word frequency. Effect estimates were extracted from models in $\mathrm{R}$ using the effects package (Fox et al., 2019). All plots were generated using the ggplot2 package (Wickham, 2009).
Table 6 illustrates another advantage of a high-powered study over less-powered ones. Most of the interactions that reached significance in the regression models fitted to the full dataset were only found to be reliable in one, two or even none of individual experiments, and are likely to go unnoticed in those studies.

\section{Power}

As indicated above, at times the results of regression models observed in the full dataset diverge from those models fitted to the data from individual experiments. An important 
Table 6 Summary of interactions of compound frequency with lexical predictors in the full dataset and across individual experiments

\begin{tabular}{|c|c|c|c|c|c|c|c|c|c|}
\hline Eye-movement variable & Predictor & Full & Study 1 & Study 2 & Study 3 & Study 4 & Study 5 & Study 6 & Study 7 \\
\hline First fixation duration & EntropyConceptRel & & & & & & & & $*$ \\
\hline Gaze duration & EntropyConceptRel & & & & $*$ & & & & \\
\hline Total fixation time & EntropyConceptRel & & & & $*$ & & & $*$ & \\
\hline First fixation duration & LeftValence & & & & & & & & \\
\hline Gaze duration & LeftValence & & & & & & & & \\
\hline Total fixation time & LeftValence & & & & & & & & \\
\hline First fixation duration & RightValence & & & & & & & & \\
\hline Gaze duration & RightValence & & & & & & & & \\
\hline Total fixation time & RightValence & & & & & & & & \\
\hline First fixation duration & LeftFamilySize & & & & & & & & \\
\hline Gaze duration & LeftFamilySize & & & & & & & & \\
\hline Total fixation time & LeftFamilySize & & $*$ & & & & & & \\
\hline First fixation duration & RightFamilySize & & & & $*$ & & & & \\
\hline Gaze duration & RightFamilySize & & & & & & & & \\
\hline Total fixation time & RightFamilySize & & & & & & & & \\
\hline First fixation duration & LeftFrequency & & & & & & & & \\
\hline Gaze duration & LeftFrequency & $*$ & & & $*$ & & & $*$ & \\
\hline Total fixation time & LeftFrequency & $*$ & & & $*$ & & & $*$ & \\
\hline First fixation duration & RightFrequency & & & $*$ & & & & & \\
\hline Gaze duration & RightFrequency & & & & & & & & \\
\hline Total fixation time & RightFrequency & & & & & & & & \\
\hline First fixation duration & CompoundLength & $*$ & & & & & & & \\
\hline Gaze duration & CompoundLength & $*$ & & & $*$ & & & & \\
\hline Total fixation time & CompoundLength & $*$ & & & $*$ & & & & \\
\hline First fixation duration & LeftST & & & $*$ & & & $*$ & & \\
\hline Gaze duration & LeftST & & $*$ & & & & & & \\
\hline Total fixation time & LeftST & & & & & & & & \\
\hline First fixation duration & RightST & & & & & & & $*$ & \\
\hline Gaze duration & RightST & & & & & & & & \\
\hline Total fixation time & RightST & & & & & & & & \\
\hline
\end{tabular}

* indicates the presence of a main effect with $t>2$ after the application of multiple comparison correction criteria

contributor to this divergence in results is the difference in statistical power originating from the fact that analyses were run across data containing different sample sizes. The aim of this section is to provide estimates of power for the effects of continuous predictors that we observed in the present study, computed across a range of sample sizes. Presently, to our knowledge there is no agreed-upon solution on how to define an effect size for a continuous predictor in a mixedeffects model. We informally defined effect size as the slope of a predictor in the model fitted to log-transformed total fixation time. This section estimates power for detecting the effect of a given magnitude (i.e., a given value of a slope) across a range of sample sizes. The modelestimated slopes for all predictors in our study are reported in Table 5: since all our predictors were $z$-transformed, these slopes represent a change in the log total fixation time per one unit of standard deviation in the predictor. As Table 5 reveals, the slopes ranged from the strongest0.117 (log CompoundFrequency) to the weakest-0.003 (EntropyConceptRel). For our simulation study of power, we based our selection of simulated slopes on effect sizes within this range, from -0.1 to -0.013 (we opted for the negative signs because most lexical effects were negative).

Figure 2 demonstrates the power curves associated with each slope. Specifically, each curve reports a power estimate for a given slope as a function of sample size. These curves were obtained from simulations, where a model was fitted to log total fixation time, with random intercepts for items and subjects and a single predictor, with a slope set to one of the values in the range of effect sizes. More often than not, the by-subject random slopes for the predictor led to models that failed to converge: for comparability, 

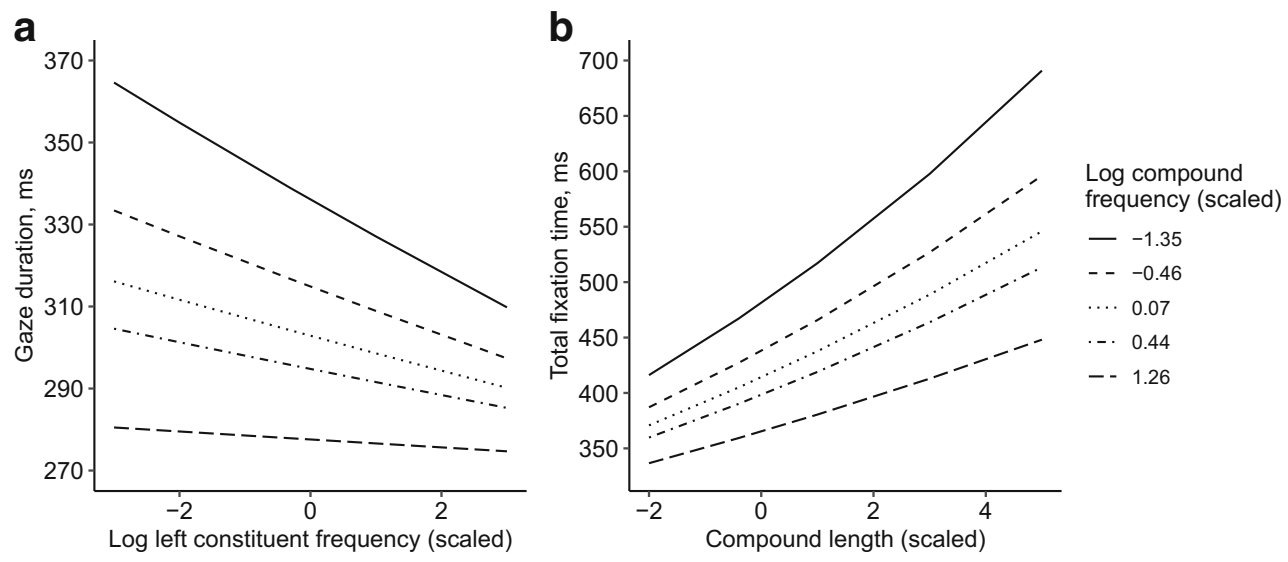

Fig. 1 a Partial effects of log left constituent frequency (scaled) modulated by log compound frequency (scaled), on gaze duration. b Partial effects of compound length (scaled) modulated by log compound frequency (scaled), on total fixation time. Slopes are provided for the $10^{\text {th }}$ (solid line), $30^{\text {th }}$ (dashed line), $50^{\text {th }}$ (dotted line), $70^{\text {th }}$ (dotdash line), and $90^{\text {th }}$ (longdash line) percentiles of the scaled values of log compound frequency (reported in the margin)

we removed this random effect from all models. Each simulation took a different sample from the total number of pooled data points, items, and subjects, and provided an inferential statistic for whether the predictor with a given slope had reached statistical significance (at the 5\% level) in the model. The percentage of models in which statistical significance had been reached indicated the power of the original model, i.e., the likelihood of detecting an effect of a given size. Random samples with different sizes were selected from the dataset used for the analysis reported above (i.e., 38,671 trials containing full sets of observations for all lexical variables). Sample sizes began at $10 \%$ of the dataset size, and increased in $10 \%$ increments up to the total number of data points in the dataset (i.e., $10 \%, 20 \% \ldots 100 \%)$. Power curves were estimated using the powercurve() function in the simr package in

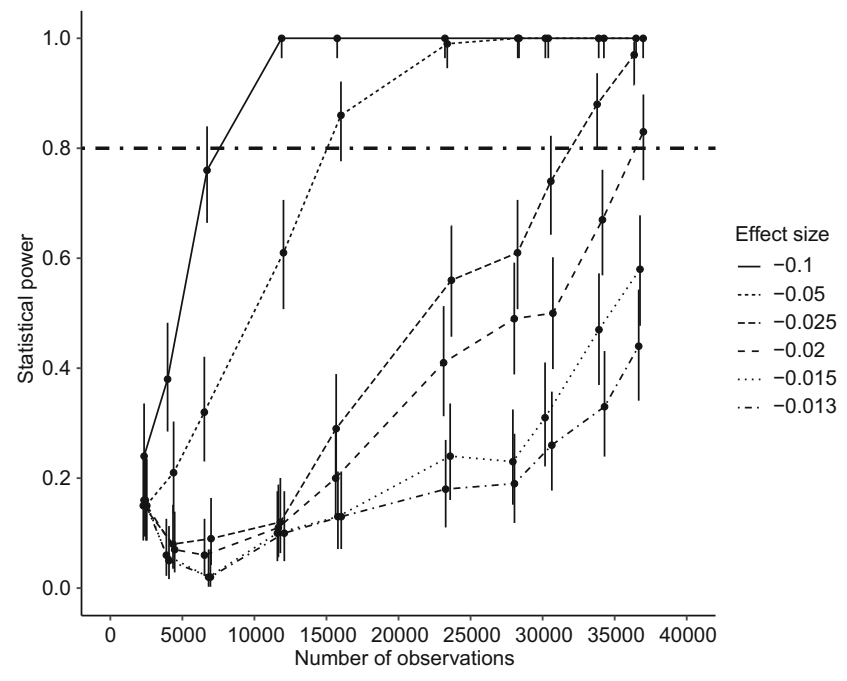

Fig. 2 Power estimates for six different regression slopes. Error bars correspond to the $95 \%$ confidence interval. Jitter added for readability
R (Green \& MacLeod, 2016), with 100 iterations per simulation and other settings set to defaults: for a detailed description of the method see Brysbaert and Stevens (2018).

As is accepted in the literature, we consider an $80 \%$ likelihood of detecting an effect at the 5\% level of statistical significance as a conventional level of power (Cohen, 2013). Figure 2 indicates that, unsurprisingly, the slope with the greatest absolute value comes with the greatest power. The slope of -0.1 -and by implication any slope with a greater absolute value-will be statistically significant at least $80 \%$ of the time in a sample of 7000 observations or greater. To link this back to our set of predictors, this suggests that the main effect of compound frequency (CompoundFrequency) on total fixation time $(-0.117)$ can be reliably estimated in a relatively small-scale eye-movement experiment, e.g., 50 participants reading 140 compounds $(50 \times 140=7000$ observations). However, effects with a slope of -0.05 require about 15,000 observations with $80 \%$ power. That is, effects on total fixation time associated with, say, compound length (CompoundLength, 0.059), and right-whole semantic transparency (RightST, -0.041) can be reliably detected in an eye-movement experiment with 50 subjects reading 300 compounds each.

Figure 2 further indicates that lexical predictors associated with slopes of approximately -0.025 (e.g., LeftST, 0.028; LeftFrequency, -0.027; and RightFrequency, -0.035) require a much larger sample size of approximately 33,000 observations to reach the nominal $80 \%$ level of power. This sample size is equivalent to an experiment with 50 participants exposed to 660 compounds, or 100 participants reading 330 compounds. An experiment of this magnitude certainly exceeds the common experimental practice and quite possibly exceeds the reasonable capacity of a mid-sized eye-tracking laboratory. Estimation of an even flatter slope of -0.02 will obtain $80 \%$ power only at 
38,000 observations, which amounts to our full dataset. Any smaller experiment will have a very high type II error rate in detecting effects of this size. Slopes with absolute values below 0.02 essentially do not stand a chance of being reliably detected even in our full dataset (which is equivalent to 50 participants reading 760 compounds). This pertains to main effects of such lexical predictors as LeftFamilySize, RightFamilySize, LeftValence, RightValence and EntropyConceptRel. The failure of this very large data set to provide adequate power strongly suggests that effect sizes in the general population are likely to be very small if they do exist. Importantly, the observed lack of statistical power to detect subtle main effects is likely to be even more drastic for interactions, because they require a greater statistical power than main effects do (Perugini et al., 2018)

Some eye-tracking studies of English compound word reading report significant main effects of semantic transparency (Juhasz et al., 2009; Schmidtke et al., 2018a; Underwood et al., 1990), while others fail to find such effects (e.g., Frisson, Niswander Klement \& Pollatsek 2008; Juhasz 2018). As discussed in Schmidtke et al. (2018a), one of the sources of inconsistency in the observation of reliable effects of semantic transparency may be the multitude of available operationalizations of semantic transparency available in the literature. Here we argue that an additional source of the inconsistency in semantic transparency effects in prior eye-movement studies may also lie in the unreliability of underpowered studies which examine this lexical characteristic. As indicated by the results of the power analysis presented here, even the two largest of prior eyemovement studies (Juhasz 2018; approximately 9000 trials before trimming) and Schmidtke et al. (2018a; 5724 data points after trimming) may not have had a sufficient amount of statistical power to reliably observe effects of semantic transparency (either RightST, 15,000 trials or LeftST, 33,000 trials). Thus, insufficient power might have been the reason why one of these individual studies found an effect while the other did not.

\section{General discussion}

This paper presents a new behavioral database of eyemovements registered during the reading of English noun-noun compounds embedded in sentences, see Data availability below for details. The database is a compilation of seven eye-tracking experiments that share a design and experimental setup but differ in stimuli and population sample. The outcome is the largest collection of eyetracking information on compounds so far that can be used for hypothesis testing through virtual experiments, i.e., experimentation without additional data collection (see Kuperman, 2015).
We supplemented the reporting of the new data with a series of analyses that estimated the influence of select lexical predictors on eye-movements to compounds. The analyses involved fitting sets of regression models, which estimated-separately-main effects of each predictor, and an interaction of each predictor (except for compound frequency) with compound frequency. These analyses revealed that the range of lexical variables that elicit robust main effects on compound processing is fairly restricted. These variables include benchmark predictors that are extremely robust in eye-movement studies (Rayner, 1998), namely, compound length and compound frequency. They also include frequencies of the compound's constituents as stand-alone words and semantic transparency (i.e., the measure of relatedness between the meanings of the compound and its morphemes). The main effects of semantic transparency corroborate the effects reported in previous eye-movement studies of English compound word reading (e.g., Juhasz et al. 2009; Schmidtke et al. 2018a; Underwood, Petley \& Clews, 1990). The results of the present database support theories in which compound word recognition benefits from the conjunctive activation of semantically related morphemes and whole words (e.g., Libben, 1998), and show that this study is sufficiently powered to observe such effects.

Furthermore, an even more restricted set of variables, i.e., compound length and left constituent frequency, showed reliable interactions with compound frequency. In all relevant models, higher-frequency compounds were associated with weaker effects of the interacting variable: i.e., a weaker positive effect of compound length and a weaker negative effect of left constituent frequency. We argue that the pattern of results suggests that compounds which are most entrenched in the reader's mental lexicon by virtue of relatively frequent exposure develop higher-quality representations of the word's orthography, phonology and semantics (Perfetti \& Hart, 2002). As a result of this enhanced lexical quality, recognition of such compounds does not benefit from semantic, formal or distributional cues as much as during the recognition of less established compounds.

Our final contribution is a power analysis, which estimated the sample sizes that are required to detect effects with magnitudes comparable to those observed in our regression models. We conducted the analyses for main effects only, but future research can address the interaction effects in much the same way using our database. Perhaps the most important finding emerging from this effort is that typical individual experiments are only equipped to detect the most robust lexical effects on compound processing. For instance, an eye-movement experiment involving up to 100-200 items and 20-30 participants will have an $80 \%$ likelihood of detecting the strongest known lexical effect, 
i.e., that of compound frequency. A sample on the order of 18,000 data points (e.g., 50 participants reading 360 compounds each) constitutes a well-powered experiment with the ability to detect an effect of compound length or semantic transparency. To reach the $80 \%$ power threshold required for the effects of constituent frequencies, the number of required observations must rise to at least 33,000 data points (e.g., 50 participants reading 660 compounds each).

Much weaker effects, which we find to be associated with a few semantic predictors, cannot be detected with the desired $80 \%$ power level, even using our full dataset of close to 40,000 observations. These results lead us to a conclusion and a recommendation for researchers. The observed data patterns identify lexical predictors that are very unlikely to have a true effect on recognition of written noun-noun English compounds during sentence reading. These are the effects that cannot be detected with a sufficient power in the present dataset, for instance, family sizes of the left and right constituents, valence of both constituents, and entropy of conceptual relations. The implication is that pursuing these effects further in smaller experiments could be a waste of research effort.

Our findings of null effects for a number of semantic variables may appear to contradict earlier work on written recognition of compounds. For instance, Juhasz and Berkowitz (2011) observed an effect of left constituent family size on gaze duration and the number of refixations in an eye-tracking experiment with 30 participants and 54 English compounds (1,620 data points before trimming), see also Bertram (2011) for a review of evidence from other languages. We believe this reported effect (along with many other effects reported in similarly small-scale studies, including our own) illustrates a well-described consequence of insufficient statistical power. Specifically, under-powered studies increase the risk of generating statistically significant but spurious (and often inflated) effects that do not reflect the true effect of a predictor (Brysbaert et al., 2016; Button et al., 2013; Ioannidis, 2005). Tables 5 and 6 further illustrate this point: many of the effects that do not reach significance in our full dataset emerge as statistically significant in individual experiments.

In sum, we believe it is possible for small-scale experiments to uncover effects that would meet the threshold of statistical reliability, yet these effects would not be true effects in the general population (these are spurious). Needless to say, the present conclusion does not necessarily apply to other languages, experimental paradigms, or morphological structures. Moreover, we don't make any claims about other types of compounding, such as Adjective-Noun or Verb-Verb compounds, or spaced English compounds. An important contribution of our power analysis is to guide choices about expending laboratory resources. Importantly, our database enables researchers to pre-test whether a lexical variable of their choice is worth examining in a separate experiment, and estimate the sample size that would be required for that experiment. This pre-test amounts to a virtual experiment in which researchers collect data, or use existing data on a lexical variable of their choice for a subset of compounds, and use the present database to (i) estimate the slope of this predictor's effect on eye-movements, and (ii) use power curves like those presented in Fig. 2 to evaluate the desired sample size for an adequately powered experiment. We would also like to note that our database affords the possibility of examining compound word reading using a design that adopts a factorial approach. For example, researchers may wish to independently obtain categorical characteristics of the compound word items contained in this database, and then assess their effects on eyemovements. Furthermore, quasi-experimental conditions may also be formed, such as by sampling high- and low-frequency words from the dataset, and then matching these words on every other lexical variable. Estimating power for factorial designs is also possible and can be found in Brysbaert and Stevens (2018) and Brysbaert (2019). However, we wish to point to out that the categorization of continuous variables and matching on variable characteristics tend to lead to a loss of statistical power (see Baayen, 2010).

\section{Conclusions}

To conclude, our paper reports that compound length, compound frequency, left constituent frequency, right constituent frequency, the semantic transparency of the left constituent and the semantic transparency of the right constituent all yield reliable effects on durational measures in the eye-movement record. Furthermore, interactions with compound frequency reveal that compounds that are likely to be more entrenched in the lexicon reveal weaker effects of length and left constituent frequency. Critically, these analyses demonstrate that effects found in smaller experiments may not hold in larger powered experiments. The publication of this corpus, which provides the largest eye-movement dataset on compound word reading so far available, will facilitate power analyses to guide future research in this area.

\section{Data availability and open practices statement}

The database files formatted as text files (.Csv) are hosted on the Open Science Framework at the following link: https://osf.io/82p6x/. When using variables that were obtained from other sources, please cite the original source. None of the experiments were preregistered. 
Acknowledgements Daniel Schmidtke, McMaster English Language Development Diploma (MELD) program, Department of Linguistics and Language, McMaster University; Julie A. Van Dyke, Haskins Laboratories, New Haven Connecticut; Victor Kuperman, Department of Linguistics and Language, McMaster University. Daniel Schmidtke's contribution was partially completed during his $\mathrm{PhD}$ studies, which was supported by the Ontario Trillium Award and a Graduate fellowship awarded by the Lewis \& Ruth Sherman Centre for Digital Scholarship (McMaster University). The remainder of Daniel Schmidtke's contribution was supported by a Post-doctoral fellowship appointment at McMaster English Language Development Diploma (MELD) programme, Faculty of Humanities, McMaster University, Canada. Victor Kuperman's contribution was partially supported by the Canadian NSERC Discovery grant RGPIN/402395-2012 415 (Kuperman, PI), the Ontario Early Researcher award (Kuperman, PI), the Canada Research Chair (Tier 2; Kuperman, PI), the SSHRC Partnership Training Grant 895-2016-1008 (Libben, PI), and the CFI Leaders Opportunity Fund (Kuperman, PI). Julie A. Van Dyke's contribution was supported by the following NIH grants to Haskins Laboratories: R01 HD-073288 (Julie A. Van Dyke, PI), and P01 HD-01994 (Jay G. Rueckl, PI).

We are thankful to Noor Al-Zanoon, Morgan Bontrager, Emma Bridgwater, Kaitlin Falkauskas, Irena Grusecki, Brooke Osborne, Sadaf Rahmanian, Katrina Reyes, Aaron So, Heidi Sarles-Whittlesey and Chloe Sukkau for data collection.

\section{References}

Acheson, D. J., Wells, J. B., \& MacDonald, M. C. (2008). New and updated tests of print exposure and reading abilities in college students. Behavior Research Methods, 40(1), 278-289.

Baayen, R. H. (2010). A real experiment is a factorial experiment. The Mental Lexicon, 5(1), 149-157.

Baayen, R. H., Piepenbrock, R., \& Gulikers, L. (1995). The CELEX lexical database (release 2). Distributed by the Linguistic Data Consortium, University of Pennsylvania.

Baayen, R. H., Kuperman, V., \& Bertram, R. (2010). Frequency effects in compound processing, (pp. 257-270). Compounding, Amsterdam/Philadelphia: Benjamins.

Balota, D., Cortese, M., Hutchison, K., Neely, J., Nelson, D., Simpson, G., \& Treiman, R. (2002). The English Lexicon Project: A webbased repository of descriptive and behavioral measures for 40,481 English words and nonwords. Washington University. Online: http://elexicon.wustl.edu..

Balota, D. A., Yap, M. J., Hutchison, K. A., Cortese, M. J., Kessler, B., Loftis, B., \& Treiman, R. (2007). The English Lexicon Project. Behavior Research Methods, 39(3), 445-459.

Benjamini, Y., \& Hochberg, Y. (1995). Controlling the false discovery rate: A practical and powerful approach to multiple testing. Journal of the Royal Statistical Society: Series B (Methodological), 57(1), 289-300.

Bertram, R. (2011). Eye movements and morphological processing in reading. The Mental Lexicon, 6(1), 83-109.

Brysbaert, M. (2019). How many participants do we have to include in properly powered experiments? A tutorial of power analysis with reference tables. Journal of Cognition, 2, 1.

Brysbaert, M., \& New, B. (2009). Moving beyond Kučera and Francis: A critical evaluation of current word frequency norms and the introduction of a new and improved word frequency measure for American English. Behavior Research Methods, 41(4), 977-990.

Brysbaert, M., \& Stevens, M. (2018). Power analysis and effect size in mixed effects models: A tutorial. Journal of Cognition, 1, 1.
Brysbaert, M., Stevens, M., Mandera, P., \& Keuleers, E. (2016). The impact of word prevalence on lexical decision times: Evidence from the Dutch Lexicon Project 2. Journal of Experimental Psychology: Human Perception and Performance, 42(3), 441.

Button, K. S., Ioannidis, J. P., Mokrysz, C., Nosek, B. A., Flint, J., Robinson, E. S., \& Munafò, M. R. (2013). Power failure: Why small sample size undermines the reliability of neuroscience. Nature Reviews Neuroscience, 14(5), 365.

Choi, W., Lowder, M. W., Ferreira, F., \& Henderson, J. M. (2015). Individual differences in the perceptual span during reading: Evidence from the moving window technique. Attention, Perception, \& Psychophysics, 77(7), 2463-2475.

Cohen, J. (2013). Statistical power analysis for the behavioral sciences. Routledge.

Cutter, M. G., Drieghe, D., \& Liversedge, S. P. (2014). Preview benefit in English spaced compounds. Journal of Experimental Psychology: Learning, Memory, and Cognition, 40(6), 1778.

De Jong, N. H., Schreuder, R., \& Harald Baayen, R. (2000). The morphological family size effect and morphology. Language and Cognitive Processes, 15(4-5), 329-365.

Ernestus, M., \& Cutler, A. (2015). BALDEY: A database of auditory lexical decisions. The Quarterly Journal of Experimental Psychology, 68(8), 1469-1488.

Falkauskas, K., \& Kuperman, V. (2015). When experience meets language statistics: Individual variability in processing English compound words. Journal of Experimental Psychology: Learning, Memory, and Cognition, 41(6), 1607-1627.

Fox, J., Weisberg, S., Adler, D., Bates, D., Baud-Bovy, G., Ellison, S., ..., et al (2012). Package 'car'. Vienna: R Foundation for Statistical Computing.

Fox, J., Weisberg, S., Friendly, M., Hong, J., Andersen, R., Firth, D., \& Fox, M. J. (2019). Package 'effects'.

Frisson, S., Niswander-Klement, E., \& Pollatsek, A. (2008). The role of semantic transparency in the processing of English compound words. British Journal of Psychology, 99(1), 87-107.

Gagné, C. L., Spalding, T. L., \& Schmidtke, D. (2019). LADEC: The large database of English compounds. Behavior Research Methods, 1-28.

Green, P., \& MacLeod, C. J. (2016). SIMR: An R package for power analysis of generalized linear mixed models by simulation. Methods in Ecology and Evolution, 7(4), 493-498.

Hyönä, J., \& Olson, R. K. (1995). Eye fixation patterns among dyslexic and normal readers: Effects of word length and word frequency. Journal of Experimental Psychology: Learning, Memory, and Cognition, 21(6), 1430.

Ioannidis, J. P. (2005). Why most published research findings are false. PLoS Medicine, 2(8), e124.

Juhasz, B. J. (2018). Experience with compound words influences their processing: An eye movement investigation with English compound words. Quarterly Journal of Experimental Psychology, $71(1), 103-112$.

Juhasz, B. J., \& Berkowitz, R. N. (2011). Effects of morphological families on English compound word recognition: A multitask investigation. Language and Cognitive Processes, 26(4-6), 653682.

Juhasz, B. J., Inhoff, A. W., \& Rayner, K. (2005). The role of interword spaces in the processing of English compound words. Language and Cognitive Processes, 20(1-2), 291-316.

Juhasz, B. J., Pollatsek, A., Hyönä, J., Drieghe, D., \& Rayner, K. (2009). Parafoveal processing within and between words. The Quarterly Journal of Experimental Psychology, 62(7), 1356-1376.

Keuleers, E., \& Balota, D. A. (2015). Megastudies, crowdsourcing, and large datasets in psycholinguistics: An overview of recent developments (Vol. 68) (8). Taylor \& Francis. 
Keuleers, E., Lacey, P., Rastle, K., \& Brysbaert, M. (2012). The British Lexicon Project: Lexical decision data for 28,730 monosyllabic and disyllabic English words. Behavior Research Methods, 44(1), 287-304.

Kim, S. Y., Yap, M. J., \& Goh, W. D. (2018). The role of semantic transparency in visual word recognition of compound words: A megastudy approach. Behavior Research Methods: 1-11.

Kuperman, V. (2013). Accentuate the positive: Semantic access in English compounds. Frontiers in Psychology, 4, 203.

Kuperman, V. (2015). Virtual experiments in megastudies: a case study of language and emotion. The Quarterly Journal of Experimental Psychology, 68(8), 1693-1710.

Kuperman, V., \& Bertram, R. (2013). Moving spaces: Spelling alternation in English noun-noun compounds. Language and Cognitive Processes, 28(7), 939-966.

Kuperman, V., Bertram, R., \& Baayen, R. H. (2008). Morphological dynamics in compound processing. Language and Cognitive Processes, 23(7-8), 1089-1132.

Kuperman, V., Schreuder, R., Bertram, R., \& Baayen, R. H. (2009). Reading polymorphemic Dutch compounds: Toward a multiple route model of lexical processing. Journal of Experimental Psychology: Human Perception and Performance, 35(3), 876.

Kuperman, V., \& Van Dyke, J. A. (2011). Effects of individual differences in verbal skills on eye-movement patterns during sentence reading. Journal of Memory and Language, 65(1), 42-73.

Kuperman, V., Estes, Z., Brysbaert, M., \& Warriner, A. B. (2014). Emotion and language: Valence and arousal affect word recognition. Journal of Experimental Psychology: General, 143(3), 1065.

Kuperman, V., Matsuki, K., \& Van Dyke, J. A. (2018). Contributions of reader-and text-level characteristics to eye-movement patterns during passage reading. Journal of Experimental Psychology: Learning, Memory, and Cognition, 44(11), 1687.

Kutner, M., Nachtsheim, C., \& Neter, J. (2004). Simultaneous inferences and other topics in regression analysis. Applied linear regression models, (4th ed.)., pp. 168-170). New York: McGrawHill Irwin.

Landauer, T. K., \& Dumais, S. T. (1997). A solution to Plato's problem: The latent semantic analysis theory of acquisition, induction, and representation of knowledge. Psychological Review, 104(2), 211-240.

Libben, G. (2006). Why study compound processing? An overview of the issues. 1-23.

Libben, G. (2014). The nature of compounds: A psychocentric perspective. Cognitive Neuropsychology, 31(1-2), 8-25.

Liversedge, S. P., Blythe, H. I., \& Drieghe, D. (2012). Beyond isolated word recognition. Behavioral and Brain Sciences, 35(5), 293-294.

Lowder, M. W., \& Gordon, P. C. (2017). Print exposure modulates the effects of repetition priming during sentence reading. Psychonomic Bulletin \& Review, 24(6), 1935-1942.

Mandera, P., Keuleers, E., \& Brysbaert, M. (2017). Explaining human performance in psycholinguistic tasks with models of semantic similarity based on prediction and counting: A review and empirical validation. Journal of Memory and Language, 92, 57-78.

Moore, M., \& Gordon, P. C. (2015). Reading ability and print exposure: Item response theory analysis of the author recognition test. Behavior Research Methods, 47(4), 1095-1109.

Morrison, R. E. (1984). Manipulation of stimulus onset delay in reading: Evidence for parallel programming of saccades. Journal of Experimental psychology: Human Perception and performance, 10(5), 667.

Perfetti, C. A., \& Hart, L. (2002). The lexical quality hypothesis. Precursors of Functional Literacy, 11, 67-86.

Perugini, M., Gallucci, M., \& Costantini, G. (2018). A practical primer to power analysis for simple experimental designs. International Review of Social Psychology, 31, 1.
Rau, A. K., Moeller, K., \& Landerl, K. (2014). The transition from sublexical to lexical processing in a consistent orthography: An eye-tracking study. Scientific Studies of Reading, 18(3), 224-233.

Rayner, K. (1998). Eye movements in reading and information processing: 20 years of research. Psychological Bulletin, 124(3), 372.

Rayner, K. (2009). Eye movements and attention in reading, scene perception, and visual search. The Quarterly Journal of Experimental Psychology, 62(8), 1457-1506.

Schmid, H. J. (2010). Does frequency in text instantiate entrenchment in the cognitive system. Quantitative Methods in Cognitive Semantics: Corpus-Driven Approaches, 101-133.

Schmidtke, D., \& Kuperman, V. (2019). A paradox of apparent brainless behavior: The time-course of compound word recognition. Cortex, 116, 250-267.

Schmidtke, D., Kuperman, V., Gagné, C. L., \& Spalding, T. L. (2016). Competition between conceptual relations affects compound recognition: The role of entropy. Psychonomic Bulletin \& Review, 23(2), 556-570.

Schmidtke, D., Van Dyke, J. A., \& Kuperman, V. (2018a). Individual variability in the semantic processing of English compound words. Journal of Experimental Psychology: Learning, Memory, and Cognition, 44(3), 421.

Schmidtke, D., Gagné, C. L., Kuperman, V., Spalding, T. L., \& Tucker, B. V. (2018b). Conceptual relations compete during auditory and visual compound word recognition. Language, Cognition and Neuroscience, 33(7), 923-942.

Schmidtke, D., Gagné, C. L., Kuperman, V., \& Spalding, T. L. (2018c). Language experience shapes relational knowledge of compound words. Psychonomic Bulletin \& Review, 25(4), 1468-1487.

Schotter, E. R., Lee, M., Reiderman, M., \& Rayner, K. (2015). The effect of contextual constraint on parafoveal processing in reading. Journal of Memory and Language, 83, 118-139.

Schramm, P., \& Rouder, J. (2019). Are reaction time transformations really beneficial? PsyArXiv March 5.

Schreuder, R., \& Baayen, R. H. (1997). How complex simplex words can be? Journal of Memory and Language, 37(1), 118-139.

Stanovich, K. E., \& West, R. F. (1989). Exposure to print and orthographic processing. Reading Research Quarterly, 402-433.

Staub, A. (2015). The effect of lexical predictability on eye movements in reading: Critical review and theoretical interpretation. Language and Linguistics Compass, 9(8), 311-327.

Staub, A., Rayner, K., Pollatsek, A., Hyönä, J., \& Majewski, H. (2007). The time course of plausibility effects on eye movements in reading: Evidence from noun-noun compounds. Journal of Experimental Psychology: Learning, Memory, and Cognition, 33(6), 1162.

Tiffin-Richards, S. P., \& Schroeder, S. (2015). Children's and adults' parafoveal processes in German: Phonological and orthographic effects. Journal of Cognitive Psychology, 27(5), 531-548.

Tomaschek, F., Hendrix, P., \& Baayen, R. H. (2018). Strategies for addressing collinearity in multivariate linguistic data. Journal of Phonetics, 71, 249-267.

Tucker, B. V., Brenner, D., Danielson, D. K., Kelley, M. C., Nenadić, F., \& Sims, M. (2018). The massive auditory lexical decision (MALD) database. Behavior Research Methods, 1-18.

Underwood, G., Petley, K., \& Clews, S. (1990). Searching for information during sentence comprehension. In Gruner, R., d'Ydewalle, G., \& Parham, R. (Eds.) From eye to mind: Information acquisition in perception, (pp. 191-203). Amsterdam.

van Heuven, W. J., Mandera, P., Keuleers, E., \& Brysbaert, M. (2014). SUBTLEX-UK: A new and improved word frequency database for British English. The Quarterly Journal of Experimental Psychology, 67(6), 1176-1190. 
von der Malsburg, T., \& Angele, B. (2017). False positives and other statistical errors in standard analyses of eye movements in reading. Journal of Memory and Language, 94, 119-133.

Warriner, A. B., Kuperman, V., \& Brysbaert, M. (2013). Norms of valence, arousal, and dominance for 13,915 English lemmas. Behavior Research Methods, 45(4), 1191-1207.
Wickham, H. (2009). ggplot2: Elegant graphics for data analysis. New York: Springer. http://ggplot2.org.

Publisher's note Springer Nature remains neutral with regard to jurisdictional claims in published maps and institutional affiliations. 